\title{
Physician Perspectives on Factors Contributing to Readmissions and Potential Prevention Strategies: A Multicenter Survey
}

Shoshana J. Herzig, MD MPH', , Jeffrey L. Schnipper, MD MPH²,3, Lauren Doctoroff, $M D^{1,2}$, Christopher S. Kim, MD, MBA 4 , Scott A. Flanders, MD4 , Edmondo J. Robinson, MD MBA ${ }^{5}$, Gregory W. Ruhnke, MD MS MPH', Larissa Thomas, MD MPH ${ }^{7,8}$, Sunil Kripalani, MD MSc 9, 10, Peter K. Lindenauer, MD MSc ${ }^{17}$, Mark V. Williams, MD ${ }^{12}$, Joshua P. Metlay, MD PhD ${ }^{13}$, and Andrew D. Auerbach, MD MPH ${ }^{14}$

'Division of General Medicine and Primary Care, Beth Israel Deaconess Medical Center, Brookline, MA, USA; ${ }^{2}$ Harvard Medical School, Boston, MA, USA; ${ }^{3}$ Hospitalist Service, Division of General Internal Medicine and Primary Care, Brigham and Women's Hospital, Boston, MA, USA; ${ }^{4}$ Division of General Medicine, University of Michigan Medical School, Ann Arbor, MI, USA; ${ }^{5}$ Value Institute and Department of Medicine, Christiana Care Health System, Wilmington, DE, USA; ${ }^{6}$ Section of Hospital Medicine, Department of Medicine, University of Chicago, Chicago, Illinois, USA; 7 Division

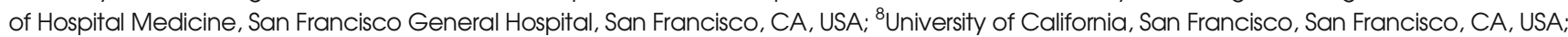
${ }^{9}$ Section of Hospital Medicine, Vanderbilt University, Nashville, TN, USA; ${ }^{10}$ Center for Clinical Quality and Implementation Research, Vanderbilt University, Nashville, TN, USA; ${ }^{11}$ Center for Quality of Care Research and Department of Medicine, Baystate Medical Center, Springfield, MA, USA; ${ }^{12}$ Center for Health Services Research, University of Kentucky, Lexington, KY, USA; ${ }^{13}$ Division of General Internal Medicine, Massachusetts General Hospital, Boston, MA, USA; ${ }^{14}$ Division of Hospital Medicine, University of California San Francisco, San Francisco, CA, USA.

BACKGROUND: The transition out of the hospital is a vulnerable time for patients, relying heavily on communication and coordination of resources across care settings. Understanding the perspectives of inpatient and outpatient physicians regarding factors contributing to readmission and potential preventive strategies is crucial in designing appropriately targeted readmission prevention efforts.

OBJECTIVE: To examine and compare inpatient and outpatient physician opinions regarding reasons for readmission and interventions that might have prevented readmission.

DESIGN: Cross-sectional multicenter study.

PARTICIPANTS: We identified patients readmitted to general medicine services within 30 days of discharge at 12 US academic medical centers, and surveyed the primary care physician (PCP), discharging physician from the index admission, and admitting physician from the readmission regarding their endorsement of pre-specified factors contributing to the readmission and potential preventive strategies.

MAIN MEASURES: We calculated kappa statistics to gauge agreement between physician dyads (PCP-discharging physician, PCP-admitting physician, and admitting-discharging physician).

KEY RESULTS: We evaluated 993 readmission events, which generated responses from 356 PCPs (36 \% of readmissions), 675 discharging physicians (68\% of readmissions), and 737 admitting physicians (74\% of readmissions). The most commonly endorsed contributing factors by both PCPs and inpatient physicians related to patient

Electronic supplementary material The online version of this article (doi:10.1007/s11606-016-3764-5) contains supplementary material, which is available to authorized users.

Received December 14, 2015

Revised May 2, 2016

Accepted May 18, 2016

Published online June 9, 2016 understanding and ability to self-manage. The most commonly endorsed preventive strategies involved providing patients with enhanced post-discharge instructions and/ or support. Although PCPs and inpatient physicians endorsed contributing factors and potential preventive strategies with similar frequencies, agreement among the three physicians on the specific factors and/or strategies that applied to individual readmission events was poor (maximum kappa 0.30).

CONCLUSIONS: Differing opinions among physicians on factors contributing to individual readmissions highlights the importance of communication between inpatient and outpatient providers at discharge to share their different perspectives, and suggests that multi-faceted, broadly applied interventions may be more successful than those that rely on individual providers choosing specific services based on perceived risk factors.

KEY WORDS: readmissions; physician opinion; survey; causes; prevention.

J Gen Intern Med 31(11):1287-93

DOI: $10.1007 / \mathrm{s} 11606-016-3764-5$

(C) Society of General Internal Medicine 2016

\section{INTRODUCTION}

Care transitions are a vulnerable time for patients, with $20 \%$ of Medicare patients readmitted within 30 days of hospital discharge. ${ }^{1}$ Despite widespread efforts using a variety of approaches for reducing readmissions, the effects of readmission reduction programs have been equivocal. ${ }^{2-5}$ In addition, debate continues on the preventability of hospital readmissions, with a systematic review estimating that as few as $27 \%$ of readmissions are truly preventable. ${ }^{6}$

The high rate of hospital readmissions and uncertain effects of readmission reduction programs may, in part, stem from incomplete understanding of the reasons for readmission or 
strategies to prevent readmission among physicians - key leaders and architects of institutional change surrounding care transitions and initiators of specific services targeted for individual patients. Physician opinions in this realm have not been investigated on a large scale. Prior studies designed to better understand reasons for readmission assessed patient perspectives on hospital readmissions but did not include physician perspectives. $^{7-9}$ Disagreement among physicians regarding factors contributing to readmissions and potential preventive strategies could contribute to poorly targeted readmission prevention efforts at both the individual patient level and the broader system level, both of which could result in limited success.

In the present study, we sought to examine agreement between primary care physicians (PCPs) and inpatient physicians regarding the major reasons for hospital readmission and regarding interventions that might have prevented the readmission in a large cohort of patients readmitted to 12 US hospitals.

\section{METHODS}

\section{Setting and Participants}

Our study is part of a collaborative effort of the Hospital Medicine Reengineering Network (HOMERuN), a national network of investigators at 12 academic medical centers (Online Supplementary Appendix). ${ }^{10}$

Patients were eligible for inclusion in our study if they were discharged from a general medicine service and had an unscheduled readmission to a general medicine service within 30 days of the index discharge. Eligible patients were readmitted between April 1, 2012, and March 31, 2013; were 18 years of age or older; and spoke English as their primary language or had a caregiver present who spoke English as their primary language. At each site, within the eligible sample, we applied a random-digit generation schema to prospectively select up to five patients per week for study participation. Patients were approached for consent in their hospital room during the readmission. If a patient declined participation, or was too sick to participate, unavailable, or otherwise unwilling after identification, the next randomly selected patient was approached for enrollment.

Following patient enrollment, we emailed or faxed a survey to each patient's PCP (when identifiable through electronic medical records), the discharging physician from the index admission based on the hospital discharge summary, and the admitting physician from the readmission based on the admission note. Only attending physicians were surveyed.

Our study was approved by the institutional review boards at the University of California-San Francisco (UCSF; the datacoordinating center) and all participating HOMERuN sites.

\section{Survey Development and Data Collection}

Surveys were developed by the HOMERuN investigators and pretested by the investigators as well as physicians not associated with the study. Successive refinements were made based on repeated rounds of feedback. The surveys to PCPs and inpatient physicians contained identical questions designed to ascertain the respondent's opinion on factors potentially contributing to the readmission as well as the probability that different types of interventions might have prevented the readmission. We asked, "In your opinion, which of the following factors may have contributed to the readmission? (CHECK ALL THAT APPLY)." Contributing factors were grouped into five major categories, based in part on a conceptual framework of the ideal transition in care, ${ }^{11}$ and respondents were asked to endorse all pre-specified individual factors that applied within each of these categories: 1) patient understanding and ability to self-manage (e.g. patient or caregiver inability to manage his/her symptoms); 2) continuity of care and provider communication (e.g. insufficient communication with post-acute care providers regarding the postdischarge plan); 3) social supports (e.g. inadequate support for non-clinical issues); 4) problems with index (initial) admission (e.g. misdiagnosis or failure to treat the condition adequately); and 5) problems with triage after index (initial) discharge (e.g. patient inappropriately went/was sent to emergency department or inappropriately readmitted from emergency department). In addition, they were asked, "How probable do you think each of [the following] potential types of interventions might have been in preventing this readmission?". Response options under each pre-specified intervention included: no probability, slightly probable, slightly less than 50-50, slightly more than 50-50, strongly probable, and nearly certain. See the Online Supplementary Appendix for full text of the surveys.

Surveys were developed and administered using the research electronic data capture (REDCap) application, an NIH-sponsored, HIPAA compliant, free secure web-based application. ${ }^{12}$ A unique survey link was emailed or faxed to each physician every 3 days until either a response was received or 14 calendar days elapsed.

Additional patient-level and admission-level data were collected via structured medical record review performed by trained research assistants.

\section{Statistical Analysis}

We report the frequency with which PCPs, discharging physicians, and readmitting physicians selected each of the prespecified factors contributing to readmission, grouped by major category (representing selection of at least one individual factor within that category) as well as by each individual factor. For strategies to prevent readmission, we report the frequency with which the three physician groups reported anything other than "no probability" for each of the potential preventive strategies (i.e. slightly probable, slightly less than $50-50$, slightly more than $50-50$, strongly probable, nearly certain). We chose this threshold because we were interested in any degree of preventability, and in order to maximize sample size for comparisons. To assess whether using a higher threshold to define endorsement of potential preventive 
strategies would lead to higher concordance levels, we performed a sensitivity analysis in which we used "slightly more than 50-50," "strongly probable," or "nearly certain" to indicate endorsement of a possible way to have prevented the readmission.

To gauge agreement among physicians on the factors contributing to and potential strategies to have prevented each individual readmission, we calculated kappa statistics for each dyad (PCP-discharging physician, $\mathrm{PCP}$-admitting physician, admitting-discharging physician) for each major category of contributing factor and each potential preventive factor. As a measure of overall agreement for provider dyads, we calculated the average kappa for each dyad across all contributing factors, and across all potential preventive strategies. All analyses were performed using SAS software version 9.2 (SAS Institute Inc., Cary, NC).

\section{Sensitivity Analysis}

To maximize sample size and make use of all available information, our main analysis included readmission events with at least one physician response (PCP, discharging, or admitting physician), and for comparisons, had non-missing data for the dyad being compared. For example, a readmission event with a missing PCP survey could still be included in the admitting and discharging physician tabulations and comparisons. Since this approach results in different patient subgroups, thus potentially influencing the results, we performed a secondary analysis in the subgroup of readmissions for which complete survey data were available (PCP, discharging, and admitting physician surveys all completed).

\section{RESULTS}

Table 1 lists the characteristics of the 993 readmitted patients included in our study. We received survey responses from 356 PCPs (36\% of readmission events), 675 discharging physicians (68\% of readmission events), and 737 admitting physicians (74\% of readmission events).

Table 2 summarizes the factors contributing to readmissions that were endorsed by PCPs, discharging physicians, and readmitting physicians. The contributing factors most commonly cited by both PCPs and inpatient physicians related to "patient understanding and ability to self-manage." Although PCPs and inpatient physicians cited the individual factors and major categories with similar frequency, agreement about their contribution to an individual readmission event was poor, with a mean kappa of 0.16 across all major categories and physician dyads (range 0.02-0.30). Levels of agreement were almost identical between provider dyads (average kappa 0.16, 0.15, and 0.16 for $\mathrm{PCP}$-discharging physician, $\mathrm{PCP}$-admitting physician, and admitting-discharging physician dyads, respectively). The highest levels of agreement between providers were seen in the category "patient understanding and ability to self-manage."
Table 1 Patient and Admission Characteristics as Documented During Index Admission $(N=993)$

\begin{tabular}{|c|c|c|}
\hline \multirow{2}{*}{$\begin{array}{l}\text { Characteristic } \\
\text { Age, mean (SD) }\end{array}$} & \multicolumn{2}{|c|}{ n $(\%)^{*}$} \\
\hline & 55 & (18) \\
\hline Married & 350 & (35) \\
\hline English as primary language & 924 & (93) \\
\hline \multicolumn{3}{|l|}{ Residence prior to admission } \\
\hline Home & 857 & (86) \\
\hline Facility $^{\dagger}$ & 59 & (6) \\
\hline Homeless/Shelter & 53 & (5) \\
\hline Other acute care hospital & 15 & (2) \\
\hline Other & 6 & (1) \\
\hline \multicolumn{3}{|l|}{ Active serious illnesses } \\
\hline Cancer & 172 & (17) \\
\hline Stage IV renal failure or hemodialysis & 126 & (13) \\
\hline Severe chronic obstructive pulmonary disease & 72 & (7) \\
\hline Hemorrhagic or ischemic stroke & 68 & (7) \\
\hline Stage III or IV congestive heart failure & 61 & (6) \\
\hline Parkinson's or other CNS degenerative disorder & 26 & (3) \\
\hline \multicolumn{3}{|l|}{ Active clinical issues at beginning of index hospitalization } \\
\hline Impaired mobility & 318 & (32) \\
\hline Nutritional impairment & 154 & (16) \\
\hline Alcohol or drug abuse & 146 & (15) \\
\hline Abnormal cognition & 115 & (12) \\
\hline Non-healing ulcer/wound & 76 & (8) \\
\hline \multicolumn{3}{|l|}{ Documentation that PCP was contacted at admission } \\
\hline Yes & 449 & (45) \\
\hline No & 460 & (46) \\
\hline No PCP or usual provider & 81 & (8) \\
\hline \multicolumn{3}{|l|}{ Discharged to } \\
\hline Home. & 800 & $(81)$ \\
\hline Facility $^{\dagger}$ & 134 & (14) \\
\hline Homeless/Shelter & 37 & (4) \\
\hline Other & 20 & (2) \\
\hline Physician discharge summary completed within $24 \mathrm{~h}$ & 779 & (79) \\
\hline
\end{tabular}

*Unless otherwise noted

${ }^{\dagger}$ Rehabilitation facility (including sub-acute rehab, acute rehab, and long-term acute care), chronic care facility (nursing home)

Physicians' three most commonly cited potential strategies for preventing readmission all involved providing patients with enhanced post-discharge instructions or support (Table 3): 1) improved self-management plan at discharge, 2) greater engagement of home and community supports, and 3) provision of resources to manage care and symptoms after discharge. Although PCPs and inpatient physicians cited potential prevention strategies with similar frequencies, agreement about the specific strategies that would be useful for an individual patient was poor, with a mean kappa of 0.13 (range $0.00-0.28$ ). Again, levels of agreement were almost identical between provider dyads (average kappa 0.14, 0.13, and 0.13 for PCP-discharging physician, PCP-admitting physician, and admitting-discharging physician dyads, respectively). Using a higher threshold to define endorsement of a strategy that may have prevented the readmission had no substantial effect on the results (Online Appendix Table 1). Agreement remained poor, and average kappa values were again similar between provider dyads $(0.03,0.10$, and 0.08 for PCP-discharging physician, PCP-admitting physician, and admitting-discharging physician dyads, respectively).

\section{Sensitivity Analysis}

After restricting our analysis to only those patients with survey data from all three physician groups $(n=155$ for contributing 
Table 2 PCP, Discharging Physician, and Readmitting Physician Perceptions Regarding Factors Contributing to Readmission*

\begin{tabular}{|c|c|c|c|c|c|c|c|c|c|}
\hline & \multicolumn{2}{|l|}{ PCP } & & \multicolumn{2}{|c|}{ Admit } & \multicolumn{3}{|c|}{ Concordance } \\
\hline & & & & & & & $\begin{array}{l}\text { PCP- } \\
\text { Disch }\end{array}$ & $\begin{array}{l}\text { PCP- } \\
\text { Admit }\end{array}$ & $\begin{array}{l}\text { Admit- } \\
\text { Disch }\end{array}$ \\
\hline & \multicolumn{2}{|c|}{$n=356$} & \multicolumn{2}{|c|}{$n=675$} & \multicolumn{2}{|c|}{$n=737$} & $n=237$ & $n=271$ & $n=516$ \\
\hline & No. & $\%$ & No. & $\%$ & No. & $\%$ & $\mathbf{k}$ & $\mathbf{k}$ & $\mathbf{k}$ \\
\hline Patient Understanding and Ability to Self-Manage & 196 & (55) & 354 & $(52)$ & 402 & (55) & 0.30 & 0.27 & 0.21 \\
\hline Patient or caregiver inability to manage his/her symptoms & 140 & (39) & 264 & (39) & 270 & $(37)$ & & & \\
\hline Patient or caregiver inability to otherwise care/provide care & 89 & $(25)$ & 124 & (18) & 154 & $(21)$ & & & \\
\hline Patient or caregiver inability to manage his/her medications & 54 & $(15)$ & 126 & (19) & 120 & (16) & & & \\
\hline $\begin{array}{l}\text { Patient or caregiver lack of understanding of the } \\
\text { post-discharge plan }\end{array}$ & 24 & (7) & 53 & (8) & 69 & (9) & & & \\
\hline Insufficient or ineffective patient or caregiver education & 22 & $(6)$ & 33 & $(5)$ & 47 & $(6)$ & & & \\
\hline Continuity of Care and Provider Communication & 132 & (37) & 169 & (25) & 200 & (27) & 0.08 & 0.02 & 0.11 \\
\hline $\begin{array}{l}\text { Inability of the patient to keep the follow-up appointment } \\
\text { or follow-up studies }\end{array}$ & 64 & $(18)$ & 65 & $(10)$ & 67 & (9) & & & \\
\hline $\begin{array}{l}\text { Insufficient communication with post-acute care providers } \\
\text { about post-discharge plan }\end{array}$ & 51 & (14) & 27 & (4) & 29 & (4) & & & \\
\hline Insufficient monitoring of the patient's condition(s) after discharge & 43 & $(12)$ & 79 & $(12)$ & 98 & $(13)$ & & & \\
\hline $\begin{array}{l}\text { Failure to obtain an appropriately timely follow-up appointment } \\
\text { or follow-up studies }\end{array}$ & 19 & $(5)$ & 41 & (6) & 37 & $(5)$ & & & \\
\hline Discharge summary unavailable in timely manner & 14 & $(4)$ & 5 & (1) & 6 & $(1)$ & & & \\
\hline $\begin{array}{l}\text { Discharge summary poorly written or with missing or } \\
\text { erroneous information }\end{array}$ & 5 & (1) & 3 & $(0)$ & 3 & $(0)$ & & & \\
\hline Problems with Index (Initial) Admission & 95 & (27) & 88 & (13) & 137 & (19) & 0.13 & 0.07 & 0.13 \\
\hline Discharged from the hospital too soon & 50 & (14) & 28 & (4) & 44 & $(6)$ & & & \\
\hline Inappropriate/inadequate treatment of the patient & 33 & (9) & 30 & (4) & 58 & (8) & & & \\
\hline No or inadequate end-of-life or goals of care planning & 16 & (4) & 28 & (4) & 26 & (4) & & & \\
\hline Misdiagnosis & 7 & (2) & 15 & (2) & 30 & (4) & & & \\
\hline Absent, erroneous, or incomplete medication reconciliation & 5 & (1) & 1 & $(0)$ & 5 & (1) & & & \\
\hline Social Supports & 80 & (22) & 94 & (14) & 120 & (16) & 0.14 & 0.14 & 0.20 \\
\hline Inadequate support for non-clinical issues & 38 & (11) & 59 & (9) & 59 & $(8)$ & & & \\
\hline Inadequate home services or equipment after discharge & 36 & $(10)$ & 27 & (4) & 51 & (7) & & & \\
\hline Inappropriate choice of discharge destination & 29 & $(8)$ & 30 & (4) & 34 & $(5)$ & & & \\
\hline Problems with Triage after Index (Initial) Discharge & 17 & (5) & 50 & (7) & 42 & (6) & 0.16 & 0.26 & 0.15 \\
\hline $\begin{array}{l}\text { Patient inappropriately went/was sent to ED or } \\
\text { inappropriately readmitted from ED }\end{array}$ & 17 & $(5)$ & 50 & (7) & 42 & (6) & & & \\
\hline Average $\mathrm{k}$ & $\mathrm{N} / \mathrm{A}$ & $\mathrm{N} / \mathrm{A}$ & $\mathrm{N} / \mathrm{A}$ & N/A & $\mathrm{N} / \mathrm{A}$ & $\mathrm{N} / \mathrm{A}$ & 0.16 & 0.15 & 0.16 \\
\hline
\end{tabular}

* Section headings (and corresponding numbers in bold) represent major categories, reflecting the selection of at least one individual sub-item Abbreviations: Disch discharging physician, Admit readmitting physician, $k$ kappa statistic

Table 3 PCP, Discharging Physician, and Readmitting Physician Perceptions Regarding Potential Strategies for Preventing Readmission

\begin{tabular}{|c|c|c|c|c|c|c|c|c|c|}
\hline & \multicolumn{2}{|l|}{$\mathbf{P C P}$} & \multicolumn{2}{|c|}{ Disch } & \multicolumn{2}{|c|}{ Admit } & \multicolumn{3}{|c|}{ Concordance } \\
\hline & & & & & & & $\begin{array}{l}\text { PCP- } \\
\text { Disch }\end{array}$ & $\begin{array}{l}\text { PCP- } \\
\text { Admit }\end{array}$ & $\begin{array}{l}\text { Admit- } \\
\text { Disch }\end{array}$ \\
\hline & \multicolumn{2}{|c|}{$n=305$} & \multicolumn{2}{|c|}{$n=642$} & \multicolumn{2}{|c|}{$n=694$} & $n=190$ & $n=212$ & $n=440$ \\
\hline & No. & $(\%)^{*}$ & $\mathbf{n}$ & $(\%)$ & $\mathbf{n}$ & $(\%)$ & $\mathbf{k}$ & $\mathbf{k}$ & $\mathbf{k}$ \\
\hline Improved self-management plan at discharge & 184 & $(60)$ & 343 & $(53)$ & 359 & $(52)$ & 0.22 & 0.15 & 0.21 \\
\hline Greater engagement of home and community supports & 177 & $(58)$ & 287 & $(45)$ & 305 & $(44)$ & 0.13 & 0.24 & 0.18 \\
\hline $\begin{array}{l}\text { Provision of resources to manage care and symptoms } \\
\text { after discharge }\end{array}$ & 177 & $(58)$ & 300 & (47) & 327 & (47) & 0.28 & 0.21 & 0.12 \\
\hline Improved discharge planning & 139 & $(46)$ & 202 & $(31)$ & 233 & (34) & 0.09 & 0.04 & 0.12 \\
\hline $\begin{array}{l}\text { Improved coordination of care between inpatient and } \\
\text { outpatient providers }\end{array}$ & 124 & $(41)$ & 209 & (33) & 254 & $(37)$ & 0.13 & 0.11 & 0.09 \\
\hline More complete communication of information & 112 & $(37)$ & 166 & $(26)$ & 209 & $(30)$ & 0.07 & 0.00 & 0.11 \\
\hline Improved attention to medication safety & 104 & (34) & 135 & (21) & 159 & (23) & 0.09 & 0.15 & 0.13 \\
\hline $\begin{array}{l}\text { Improved clarity, timeliness, or availability of } \\
\text { information provided at discharge }\end{array}$ & 99 & (32) & 138 & $(22)$ & 164 & (24) & 0.13 & 0.10 & 0.07 \\
\hline Average $\mathrm{k}$ & $\mathrm{N} / \mathrm{A}$ & N/A & N/A & N/A & $\mathrm{N} / \mathrm{A}$ & N/A & 0.14 & 0.13 & 0.13 \\
\hline
\end{tabular}

Abbreviations: Disch discharging physician, Admit readmitting physician, $k$ kappa statistic

* "No. (\%)" represents the number reporting anything other than "no probability" of preventing readmission (i.e. slightly probable, slightly less than 50-50, slightly more than 50-50, strongly probable, nearly certain) 
factors, $n=138$ for potential preventive strategies), our results were similar (Online Appendix Table 2 and Online Appendix Table 3). Agreement remained poor, and average kappa values were again almost identical between provider dyads for contributing factors $(0.22,0.21$, and 0.24 for PCP-discharging physician, $\mathrm{PCP}$-admitting physician, and admitting-discharging physician dyads, respectively) and preventive strategies $(0.13,0.11$, and 0.11 for $\mathrm{PCP}-$ discharging physician, $\mathrm{PCP}$-admitting physician, and admitting-discharging physician dyads, respectively).

\section{DISCUSSION}

In this large national study of PCP and inpatient physician opinions on readmission within 30 days of discharge, the contributing factors most commonly endorsed by both PCPs and inpatient physicians related to patient understanding of and ability to self-manage their illness. Similarly, the most commonly endorsed potential preventive strategies involved providing patients with enhanced post-discharge instructions and/or support. However, when examined within individual patients, agreement as to the specific factors and strategies that applied was poor. Our results highlight the importance of communication between inpatient and outpatient providers at the time of discharge, in order to ensure that all vantage points are represented in the development of readmission prevention strategies for a given patient. Our findings also suggest that multifaceted, broadly applied interventions may be more successful than those that rely on individual providers choosing specific services based on perceived risk factors for a particular patient.

A prior study demonstrated that inpatient physicians were unable to accurately predict which patients would be readmitted. ${ }^{13}$ Our study extends these findings, suggesting that not only are physicians unable to predict who will be readmitted, but physicians caring for the same patient have different views on why that patient was readmitted and on actions that could have prevented the readmission. While our study did not investigate the reasons for these differing views, it seems likely that some of the observed differences reflect the differing vantage points of inpatient and outpatient physicians. However, the fact that discordant views were just as prevalent between inpatient physicians as they were between PCPs and inpatient physicians suggests that our findings are not solely reflective of the differing vantage points across the inpatientoutpatient divide. Our findings may reflect the lack of a clear and objective answer regarding what caused a readmission in most cases, as well as the multifactorial nature of readmissions. Different opinions by different providers may also be due to common cognitive biases such as confirmation bias, availability heuristic, focusing effect, and outcome bias.

Regardless of the reason for the differing viewpoints, when taken together, these findings emphasize the importance of communication between inpatient and outpatient providers at the time of discharge, and of incorporating multiple vantage points when designing interventions to prevent readmission; each provider views the transition through a different lens, and interventions that fail to incorporate these differing perspectives may have limited impact. However, our results also underscore the difficulty in developing effective and individually targeted prevention strategies - even after incorporating multiple provider perspectives - when the key causes of readmissions are not agreed upon. Lack of agreement weakens our ability to develop a valid understanding of the key factors contributing to readmissions, and accordingly, limits the development of successful preventive approaches, particularly if they are too patient-specific. A possible corollary is that multifaceted, broadly applied interventions may be more successful than those that rely on individual providers choosing specific services based on perceived risk factors for a particular patient. These ideas are supported by a recent systematic review of randomized trials to prevent readmissions, in which interventions with many components and those involving more individuals in care delivery were found to be significantly more effective than other interventions. ${ }^{14}$

Our finding that both PCPs and inpatient physicians cited patient-related factors and prevention strategies more commonly than physician- or system-oriented factors or strategies (e.g. problems relating to the initial admission, improved attention to medication safety) suggests a patient-focused view on why readmissions happen and how they can be prevented. While this could reflect the inherent difficulty in recognizing the flaws of the systems in which we are enmeshed and the tendency to assign fault to others, it could also reflect physician belief that we are not yet delivering sufficiently patientcentered care in this realm. While we may disagree on specific ways to prevent a given readmission, our results do suggest that physicians-PCPs and inpatient physicians alike-believe that a large portion of readmissions could be prevented by strategies focused on enhancing patient understanding and the ability to self-manage with help from home and community supports. Recent studies support these physician beliefs: a systematic review found that strategies supporting patient capacity for self-care and providing comprehensive postdischarge support to patients and caregivers were more effective at preventing readmissions than were other interventions, and a recent analysis of hospitalized adult medical patients in an urban academic safety-net hospital demonstrated that patients with lower levels of patient activation had a higher rate of 30-day hospital readmission. ${ }^{14,15}$ In addition, a recent cohort study investigating predictors of early versus late readmissions demonstrated that social determinants of health, including barriers to learning, were significantly associated with readmissions, regardless of timing. ${ }^{16}$ Developing and testing broad-based strategies to enhance patient activation and patient capacity for self-care is a potential target of future research. Utilizing an objective risk assessment tool such as the Patient Activation Measure could help identify the patients who stand to benefit most from such interventions. ${ }^{17}$

Our study has several strengths, including the large multicenter design, with data collection at the time of the 
readmission, and the inclusion of multiple physician viewpoints. There are also several limitations. First, because we focused on general medicine patients readmitted to general medicine services at academic hospitals, our results may not be generalizable to non-academic hospitals or patients discharged by or readmitted to other services. It is possible that levels of physician agreement would be different when focusing on patients readmitted to other services. Furthermore, we did not include advanced practice providers or the physicians of patients who speak languages other than English, and our results therefore may not be generalizable to these groups. In addition, although the inpatient physician response rate was high, the PCP response rate was low, and thus these responses may not be representative of the population of PCPs from which they were drawn.

The fact that our survey has not been validated is an additional limitation. Although we included a comprehensive list of factors and preventive measures based on a conceptual framework of the ideal transition in care, ${ }^{11}$ our findings are limited to those domains included in our categorization scheme. While the validity of this approach is partially supported by the finding that readmission reduction programs including more domains from this framework had significantly higher effectiveness, it is nonetheless possible that this categorization scheme failed to sufficiently capture some of the true contributors to readmission. ${ }^{4}$ We also did not test inter-rater reliability, test-retest reliability, parallel-forms reliability, or internal consistency reliability of our survey instrument, a limitation which may have contributed to failure to accurately or reproducibly measure the constructs in which we were interested. While gaps in psychometric testing of our surveys may have limited accuracy in factor identification by individual physicians, it is less clear how this limitation might have effected physicians differentially and, in turn, how and whether it might have impacted our findings regarding concordance of viewpoints, particularly when physicians did not appear to have difficulty in identifying at least one contributor in each case. Finally, the contributors and strategies identified by physicians in our study are based on their own perceptions and may not accurately reflect the true contributors to or strategies for preventing readmission.

A final limitation relates to the fact that kappa is influenced by the prevalence of an item. For example, in situations where physicians chose a particular readmission contributor infrequently, chance agreement is high and kappa is accordingly reduced. While this "penalty" is felt by many to be appropriate, ${ }^{18-20}$ we attempted to minimize this phenomenon by reporting kappa values only for aggregate categories of factors predicting readmissions. The fact that kappa was generally low even for highly prevalent selections argues that the prevalence phenomenon does not explain the low rates of agreement we observed.

In conclusion, in this multicenter study on readmissions to general medicine services within 30 days of hospital discharge, we found that inpatient and outpatient physicians shared a common belief that interventions aimed at enhancing patient understanding and ability to self-manage may be most effective in preventing readmissions. The differing physician opinions that we observed regarding factors contributing to individual readmissions highlights the importance of communication between inpatient and outpatient providers at the time of discharge in order to share their different viewpoints, and suggests that multifaceted, broadly applied interventions may be more successful than those that rely on individual providers choosing specific services based on perceived risk factors for a particular patient.

Author Contributions: Dr. Herzig had full access to all of the data in the study, and takes responsibility for the integrity of the data and the accuracy of the data analysis.

Study Concept and Design: All authors.

Acquisition of Data: All authors.

Analysis and Interpretation of Data: All authors.

Drafting of the Manuscript: Herzig, Doctoroff.

Critical Revision of the Manuscript for Important Intellectual Content: All authors.

Statistical Analysis: Herzig.

Study Supervision: Herzig, Auerbach.

Corresponding Author: Shoshana J. Herzig, MD MPH; Division of General Medicine and Primary CareBeth Israel Deaconess Medical Center, 1309 Beacon Street, 2nd Floor, Brookline, MA 02446, USA (e-mail: sherzig@bidmc.harvard.edu).

\section{Compliance with Ethical Standards:}

Funding: The study was funded through an unrestricted research grant from the American Association of Medical Colleges, and in part by 2 UL1 TR000445-06 from the National Center for Advancing Translational Sciences. Dr. Auerbach was supported by NHLBI K24HL098372, and Dr. Herzig was supported by NIA K23AG042459. The funding organizations had no role in the design or conduct of the study; collection, management, analysis, or interpretation of the data; preparation, review, or approval of the manuscript; or decision to submit the manuscript for publication.

Conflict of Interest: The authors declare that they have no conflict of interest.

\section{REFERENCES}

1. Jencks SF, Williams MV, Coleman EA. Rehospitalizations among patients in the Medicare fee-for-service program. N Engl J Med. 2009;360(14):14181428.

2. Rennke S, Nguyen OK, Shoeb MH, Magan Y, Wachter RM, Ranji SR. Hospital-initiated transitional care interventions as a patient safety strategy a systematic review. Ann Intern Med. 2013;158(5_Part_2):433440

3. Hansen LO, Young RS, Hinami K, Leung A, Williams MV. Interventions to reduce 30-day rehospitalization: a systematic review. Ann Intern Med. 2011;155(8):520-528.

4. Burke RE, Guo R, Prochazka AV, Misky GJ. Identifying keys to success in reducing readmissions using the ideal transitions in care framework. BMC Health Serv Res. 2014; 14:423

5. Kripalani S, Theobald CN, Anctil B, Vasilevskis EE. Reducing hospital readmission rates: current strategies and future directions. Annu Rev Med. 2014;65:471-485. 
6. van Walraven C, Bennett C, Jennings A, Austin PC, Forster AJ. Proportion of hospital readmissions deemed avoidable: a systematic review. CMAJ : Can Med Assoc J = J Assoc Med Can. 2011;183(7):E391-402.

7. Cawthon C, Walia S, Osborn CY, Niesner KJ, Schnipper JL, Kripalani S. Improving care transitions: the patient perspective. J Health Commun. 2012;17(Suppl 3):312-324.

8. Cain CH, Neuwirth E, Bellows J, Zuber C, Green J. Patient experiences of transitioning from hospital to home: an ethnographic quality improvement project. J Hosp Med. 2012;7(5):382-387.

9. Kangovi S, Grande D, Meehan P, Mitra N, Shannon R, Long JA Perceptions of readmitted patients on the transition from hospital to home. J Hosp Med. 2012;7(9):709-712.

10. Auerbach AD, Patel MS, Metlay JP, et al. The Hospital Medicine Reengineering Network (HOMERuN): a learning organization focused on improving hospital care. Acad Med : J Assoc Am Med Coll. 2014;89(3):415-420.

11. Burke RE, Kripalani S, Vasilevskis EE, Schnipper JL. Moving beyond readmission penalties: creating an ideal process to improve transitional care. J Hosp Med. 2013;8(2):102-109.

12. Harris PA, Taylor R, Thielke R, Payne J, Gonzalez N, Conde JG. Research electronic data capture (REDCap)-a metadata-driven methodology and workflow process for providing translational research informatics support. J Biomed Inform. 2009;42(2):377-381.
13. Allaudeen N, Schnipper JL, Orav EJ, Wachter RM, Vidyarthi AR. Inability of providers to predict unplanned readmissions. J Gen Intern Med. 2011;26(7):771-776

14. Leppin AL, Gionfriddo MR, Kessler M, et al. Preventing 30-day hospital readmissions: a systematic review and meta-analysis of randomized trials. JAMA Intern Med. 2014;174(7):1095-1107.

15. Mitchell SE, Gardiner PM, Sadikova E, et al. Patient activation and 30day post-discharge hospital utilization. J Gen Intern Med. 2014;29(2):349355.

16. Graham KL, Wilker EH, Howell MD, Davis RB, Marcantonio ER. Differences between early and late readmissions among patients: a cohort study. Ann Intern Med. 2015;162(11):741-749.

17. Hibbard JH, Mahoney ER, Stockard J, Tusler M. Development and testing of a short form of the patient activation measure. Health Serv Res. 2005;40(6 Pt 1):1918-1930.

18. Cicchetti DV, Feinstein AR. High agreement but low kappa: II. Resolving the paradoxes. J Clin Epidemiol. 1990;43(6):551-558.

19. Hoehler FK. Bias and prevalence effects on kappa viewed in terms of sensitivity and specificity. J Clin Epidemiol. 2000;53(5):499-503.

20. Sim J, Wright CC. The kappa statistic in reliability studies: use, interpretation, and sample size requirements. Phys Ther. 2005;85(3):257268. 